\title{
Platelet hyperactivity and fibrin clot structure in transient ischemic attack individuals in the presence of metabolic syndrome: a microscopy and thromboelastography study
}

\author{
Mia-Jeanne van Rooy ${ }^{1}$, Wiebren Duim² ${ }^{2}$ Rene Ehlers ${ }^{3}$, Antoinette V. Buys ${ }^{4}$ and Etheresia Pretorius ${ }^{1 *}$ id
}

\begin{abstract}
Background: Strokes are commonly preceded by transient ischemic attacks (TIAs). TIA is often associated with metabolic syndrome (causing chronic inflammation), resulting in a proinflammatory- and procoagulantenvironment. The aim of this study was to determine whether platelet- and fibrin network-morphology or coagulation profiles of individuals that suffered a TIA in the presence of metabolic syndrome was altered when compared to healthy individuals.
\end{abstract}

Materials and methods: The study consisted of 40 voluntary participants. Twenty individuals that suffered a TIA in the previous $48 \mathrm{~h}$ with at least two metabolic syndrome risk factors present and twenty healthy age-matched controls. Scanning electron- and atomic force microscopy was used to study platelet- and fibrin-morphology, atomic force microscopy was used to study platelet- and fibrin fiber-elasticity and thromboelastography for the study of coagulation profiles. Statistical analysis was performed to compare the two groups. In all cases a p-value of less than 0.05 was considered statistically significant.

Results: Platelets of the control group appeared spherical with few pseudopodia present while the platelets of the TIA individuals presented with numerous pseudopodia and spreading, indicating activation. Platelet aggregation was also present. The fibrin networks of the healthy individuals consist of thick and thin fibers that form an organized network of fibers. The fibrin networks of the TIA individuals appeared less organized with less taut fibers. Fibrin fiber thickness was found to be significantly increased in the TIA group ( $p$-value $<0.001$ ) when compared to healthy controls. The thicker fibers formed irregular networks with thick masses of fibrin fibers. Platelet and fibrin fiber elasticity was found to be significantly lower in the experimental group ( $p$-value 0.0042 and $p$-value 0.0007 respectively). The hemostatic profiles of the diseased individuals did not differ significantly ( $p$-value $>0.05$ ) from the healthy controls, indicating a normal functioning coagulation cascade.

Conclusion: The findings indicate that pathological clot formation is not caused by alterations in the coagulation cascade but rather by the premature activation of platelets (as a result of chronic inflammation) that in turn causes altered fibrin formation.

Keywords: Transient ischemic attack, Coagulation, Platelets, Fibrin network, Metabolic syndrome, Chronic inflammation, Electron microscopy, Thromboelastography

\footnotetext{
*Correspondence: resia.pretorius@up.ac.za

'Department of Physiology, Faculty of Health Sciences, University of Pretoria, Private Bag ×323, Arcadia 0007, South Africa

Full list of author information is available at the end of the article
} 


\section{Background}

Ischemic heart disease and stroke accounts for $25 \%$ of deaths in the world every year and in the USA 240000 people suffer a transient ischemic attack (TIA) annually $[1,2]$. TIA is defined as: "A brief episode of neurologic dysfunction caused by focal brain or retinal ischemia, with clinical symptoms typically lasting less than one hour and without evidence of acute infarction" [3, 4]. TIAs, similar to angina, is usually an ominous predictor of future vascular complications [5]. The likelihood of suffering a stroke after a TIA is higher than suffering a recurrent stroke after a first stroke, indicating that TIAs should be treated with the utmost care in order to avoid possible events that may have severe neurological consequences [5]. Although several physical characteristics (such as age) can increase the likelihood of an ischemic event occurring [1], metabolic syndrome is currently the most prominent risk factor associated with these events [6]. For this manuscript the National Cholesterol Education Program - Adult Treatment Panel III (NCEP ATP III) definition was employed since this definition links the pro-thrombotic- and proinflammatory-state seen with metabolic syndrome and thrombotic events. Furthermore since no definite conclusion has been reached regarding criteria that should be absolutely required, this definition circumvents this controversy by suggesting that all risk factors play a role in the development of metabolic syndrome and its complications [7].

Metabolic syndrome is linked to chronic inflammation another major risk factor for the development of ischemic events, since inflammation is linked to alterations in coagulation $[6,8]$. For a review on blood coagulation in normal physiology and specifically platelet action see one of the following [6, 9-11]. Important to mention for this study is that fibrin and platelets are the major role players in both the elasticity and rigidity of the formed clot [12]. The elastic properties of fibrin and platelets allow clot deformation to occur under normal physiological conditions, but also influence the rigidity of the clot. Since fibrin fibers are stiffer when stretching than when bending, the elasticity of the clot is dependent on the cross-linking of the fibers as well as the interaction between the fibers and platelets $[13,14]$. Once fibrin fibers and platelets have formed the initial clot, platelet contraction occurs that decreases the size of the clot (retraction) and also places strain on the fibrin fibers, thereby altering fibrin fiber rigidity as well as organisation of the fibers [12].

In the presence of inflammation, normal physiological coagulation is altered when the inflammatory mediators modify the endothelium in the damaged area to become proinflammatory and pro-thrombotic [9]. Importantly inflammation is further associated with thrombosis since coagulation is stimulated by inflammation and inflammation is enhanced in the presence of procoagulant activity [15-17]. Inflammation influences coagulation by increasing the production of coagulation proteins, reducing the activity of the anticoagulant pathway and by preventing fibrinolysis $[17,18]$. Together these alterations could lead to the formation of pathological thrombi resulting in infarcts in the heart or brain. Although atherosclerosis may be one cause of TIA, the presence of metabolic syndrome could affect the coagulation system in some way, leading to a TIA even before atherosclerosis develops.

Since a large number of individuals that are affected by metabolic syndrome suffer cerebral ischemic events, finding new therapeutic targets by elucidating new factors that contribute to these incidents are crucial to the treatment and prevention of the events. This manuscript consequently focuses on the alterations of the coagulation system in TIA individuals in the presence of the metabolic syndrome. Special attention was paid to platelets and fibrin networks and the effect on the overall hemostatic profile of the participants using scanning electron- (SEM) and atomic force-microcopy (AFM) as well as thromboelastography (TEG).

\section{Materials and methods}

\section{Sample selection}

Twenty voluntary participants (between the ages of 39 and 78) that suffered a TIA in the presence of the risk factors associated with metabolic syndrome using the NCEP ATP III criteria were included in this study as experimental group, along with 20 healthy, age-matched individuals that served as the control group. The Human Ethics Committee of the University of Pretoria granted ethical clearance for this study. A qualified neurologist made the diagnosis of TIA after several diagnostic (e.g. magnetic resonance imaging) and laboratory-based blood tests (e.g. platelet count and coagulation parameters) were performed. Blood samples, via venipuncture, for this study were collected after informed consent was obtained from all participants. Blood was drawn from patients within $48 \mathrm{~h}$ after the TIA attack to ensure that the acute phase of the attack was studied. Several inclusion and exclusion criteria were used to determine eligibility for this study.

\section{Experimental group}

Inclusion criteria:

- A definite diagnosis of TIA (any uncertainty regarding diagnosis resulted in ineligibility for participation in the study)

- Males and females over the age of 18 years

- At least two metabolic syndrome symptoms present when the TIA occurred 


\section{Exclusion criteria:}

- Smoking

- Heavy drinking as indicated in patient history that could confound inflammatory effects

- Hormone replacement therapy

- Known common inflammatory conditions such as asthma, human immunodeficiency virus (HIV) or tuberculosis not related to the risk factors associated with metabolic syndrome

- Treatment with tissue plasminogen activator directly after the event to prevent any confounding of results regarding the coagulation profile

Since metabolic syndrome is a pre-diabetic condition, diabetes was not excluded in this study.

Of the 20 experimental participants all 20 were viscerally obese, 17 had diagnosed hypertension, 18 hypercholesterolemia and 12 a high fasting glucose. No carotid stenosis was detected in any of the participants with magnetic resonance angiography or with echocardiography of the carotid arteries. Two individuals had subclinical calcified plaque present in the carotid arteries, 2 had an increased intima media thickness unilaterally and 2 bilaterally. Only 1 patient was diagnosed with atherosclerosis. Two individuals had valve disease, most likely as a result of hypertension, 1 had left ventricular hypertrophy and 3 patients tachycardia. Although tachycardia had been diagnosed at the time of the attack the electrocardiogram (ECG) of 1 patient indicated tachycardia, the other 2 had normal ECG recordings due to effective treatment. A careful inventory of chronic medication or treatments after the attack was taken to determine whether the medication could affect the results of this study. The medications with known effects on coagulation are summarized in Table 1 . Since all the patients were either on anticoagulants prior to the attack or treated with anticoagulants and/or antiplatelet medication after the attack, these drugs could decrease the coagulation potential of the individual. Since the findings of this study could be blunted by the presence of the drugs, the significant findings of the study regardless of the presence of anticoagulants or antiplatelet therapy makes these findings even more important. For this reason the use of anticoagulants or antiplatelet medication was not used as an exclusion criterion in this study.

\section{Healthy group \\ Inclusion criteria:}

- Over the age of 18 years

- No inflammatory conditions

Exclusion criteria:

- Smoking

- Heavy drinking

- Chronic diseases or medication use

- Hormone replacement therapy or other medication that could affect coagulation

- Any risk factors associated with metabolic syndrome

\section{Scanning electron microscopy}

SEM was used to study platelet- and fibrin fibermorphology as well as to determine fibrin fiber thickness in both groups. Platelet- and fibrin network-morphology were studied by preparing platelet-rich plasma (PRP). PRP was prepared by drawing $5 \mathrm{ml}$ of blood in a sodium citrate vacuum tube $(3,8 \%$ final concentration: BD Vacutainer). The blood sample was centrifuged at

Table 1 Medication and the effect on coagulation

\begin{tabular}{|c|c|c|c|c|}
\hline Medication & Nr of patients & Medication administered & Comment & Ref \\
\hline Anticoagulant & 4 & Warfarin, Xarelto, Clexane & Decrease production of coagulation factors by the liver. & {$[41]$} \\
\hline Cox-1 inhibitor & 12 & Ecotrin, Disprin CV, Disprin & $\begin{array}{l}\text { Inhibits cox-1 activity thereby preventing the production } \\
\text { of } \mathrm{TXA}_{2} \text { and platelet aggregation }\end{array}$ & {$[42]$} \\
\hline $\mathrm{P} 2 \mathrm{Y}_{12}$-inhibitor & 5 & Plavix (Clopidogrel) & $\begin{array}{l}\text { Prevents release of ADP and thereby the activation of } \\
\text { platelets and subsequent platelet aggregation }\end{array}$ & {$[43]$} \\
\hline$\beta$-blocker & 6 & $\begin{array}{l}\text { Adco-biscor, Bilocor, Tenbloka, } \\
\text { Bisoprolol, Hypotone, Carloc }\end{array}$ & $\begin{array}{l}\text { Down regulates } \mathrm{TXA}_{2} \text { production, but not platelet } \\
\text { aggregation and therefore not significant effect } \\
\text { on coagulation }\end{array}$ & [43] \\
\hline Decreased fasting glucose agent & 4 & $\begin{array}{l}\text { Arrow metformin, Glucophage, } \\
\text { Galvus, Starlix }\end{array}$ & $\begin{array}{l}\text { Decreases the amount of PAI-1 available, thereby } \\
\text { influencing fibrinolysis }\end{array}$ & {$[44]$} \\
\hline Synthetic insulin & 1 & Levemir & $\begin{array}{l}\text { Hyperinsulinaemia may decrease fibrinolysis, so } \\
\text { treatment can normalise fibrinolytic activity }\end{array}$ & {$[45]$} \\
\hline GABA derivatives & 2 & Nootropil & $\begin{array}{l}\text { Appears to have an antithrombotic effect, } \\
\text { but mechanism not elucidated as yet }\end{array}$ & [46] \\
\hline
\end{tabular}


$3600 \times \mathrm{g}$ for $2 \mathrm{~min}$. All SEM samples were prepared on $10 \mathrm{~mm}$ glass coverslips.

Ten $\mu$ l of PRP was used to study platelet morphology and $10 \mu \mathrm{l}$ of PRP was mixed with $5 \mu \mathrm{l}$ of human thrombin to study fibrin network morphology. Since red blood cells that are incorporated into clots have been found to alter the thickness of fibrin fibers, PRP was used to study fibrin thickness. The South African National Blood Service (SANBS) supplied human thrombin. The thrombin solution was at a concentration of $20 \mathrm{U} / \mathrm{ml}$ and was made up in a biological buffer containing $0.2 \%$ human serum albumin. When thrombin is added to the freshly prepared human PRP or whole blood, the fibrinogen is converted to fibrin and other intracellular platelet components are released into the coagulum. These components include, but are not limited to transforming growth factor, plateletderived growth factor and fibroblastic growth factor. The glass coverslips were incubated at $37{ }^{\circ} \mathrm{C}$ for $10 \mathrm{~min}$. After incubation the cover slips were placed in phosphate buffer (PB) solution on a shaker and washed for $20 \mathrm{~min}$. The wash step was included to remove any blood proteins that may be entangled in the formed blood clot. The samples were then fixed for $30 \mathrm{~min}$ in a mixture of 2,5 \% gluteraldehyde and 2,5\% formaldehyde solution. After the fixation step the samples were washed three times in PB solution for $3 \mathrm{~min}$ to remove any residual fixative. The samples were then fixated a second time in $1 \%$ osmium tetroxide $\left(\mathrm{OsO}_{4}\right)$ for $15 \mathrm{~min}$. This step was followed by another washing step, which included three washes for $3 \mathrm{~min}$ in PBS. The samples were then serially dehydrated in $30 \%$, $50 \%, 70 \%, 90 \%$ and finally three times in $100 \%$ ethanol. The samples were dried using hexamethyldisilazane (HMDS), and mounted and coated with carbon. Once the samples were coated they were examined using a SEM (Zeiss ULTRA plus FEG SEM).

It has been established that fibrin networks, and therefore blood clots consist of thick and thin fibrin fibers $[19,20]$. In order to determine whether changes in fibrin fiber thickness were present when comparing healthy and metabolic syndrome TIA individuals, 50 random fibrin fibers were measured for each participant on SEM micrographs using ImageJ (Version 1,74i, Java).

\section{Atomic force microscopy}

\section{Sample preparation}

AFM was used to study the elastic properties of the platelets and fibrin fibers of the two groups. The preparation of platelets and fibrin networks for AFM is identical to that of SEM, except after drying the samples with HMDS; the samples were not coated with carbon. The samples were left to air dry before being analyzed.

\section{AFM measurements and imaging}

Topographic images were obtained to compare the morphology of the cells and fibers studied for AFM to that of the SEM. The images were obtained by using an AFM (Dimension Icon, Bruker, USA). AFM was performed in PeakForce ${ }^{\mathrm{Tm}}$ QNM $^{\mathrm{Tm}}$ (Quantitative Nanomechanical Property Mapping) mode. Peakforce ${ }^{\mathrm{mx}}$ $\mathrm{QNM}^{\mathrm{su}}$ is similar to that of classic tapping where the amplitude oscillation is kept constant, but differs in that the maximum force applied by the probe was controlled [21].

\section{Membrane deformability (elasticity)}

Deformability (elasticity) measurements were performed on the platelets and fibrin networks of the healthy individuals and patients in the experimental group to determine whether nano-mechanical property differences were present. A rapid force-distance curve was recorded at each pixel. Calibration of the cantilever's deflection sensitivity and spring constant allowed the rapid quantitative analysis of these force-distance curves on a number of different areas on the sample. The curve obtained was used to calculate Young's modulus and also to form adhesion images (between the slope of the curve and the minimum point of the curve). Deformation is calculated using the variation between zero and the maximum force applied. Energy dissipation can be calculated by determining the area between the approach and the development of the retract curve [22-24]. Different silicon nitride cantilevers (Bruker, USA) and settings were used for the measurement of each of the specific components investigated (See Table 2).

Ten cells or areas in the case of fibrin from each patient were studied. A $500 \mathrm{~nm}$ by $500 \mathrm{~nm}$ area was scanned by performing 128 by 128 data points of the individual force curve measurements. NanoScope Analysis (Version R3, Bruker, USA) was used to analyze 50 randomly selected force curves to fit to the modulus model to the unloading portion of the curve. For this study the Derjaguin-Muller-Toporov Model was used for the determination of Young's Modulus by fitting the slope of the obtained force-distance curve to the model [25]. A curve was only incorporated into the final modulus measurements if the goodness of fit of the data curve to the modulus model was above 0.85 . The goodness of fit is determined between the model and the acquired data curve by calculating the ratio of explained variation to total variation in the data set. Young's modulus is used to gauge the rigidity of the membrane that is measured. The rigidity is representative of the elasticity or deformability of the cell membrane and is usually defined by the stress divided by the strain on the specific membrane. Higher values indicate decreased deformability, 
Table 2 Cantilevers and cantilever parameters (nominal values)

\begin{tabular}{llllll}
\hline Component investigated & Probe name & Spring constant $(\mathrm{N} / \mathrm{m})$ & Resonant frequency & Tip radius & Peak force $(\mathrm{nN})$ \\
\hline Fibrin Fibers & OTESPA & $12-103$ & $345-361 \mathrm{kHz}$ & $15 \mathrm{~nm}$ & 150 \\
Platelet & ScanAsyst & 0.4 & $70 \mathrm{kHz}$ & $8 \mathrm{~nm}$ & 4 \\
\hline
\end{tabular}

which is highly relevant to the normal functioning of red blood cells, platelets and fibrin networks.

\section{Thromboelastography}

TEG ${ }^{\circ}$ was utilized to study the hemostatic profiles of the participants. Whole blood of the participants was obtained in citrate tubes. The blood was centrifuged at $300 \times \mathrm{g}$ for $10 \mathrm{~min}$ to obtain PRP. The PRP was stored in $500 \mu \mathrm{l}$ aliquots in $\mathrm{a}-70{ }^{\circ} \mathrm{C}$ freezer. On the day of experimentation, the aliquots were removed from the freezer and rapidly thawed at room temperature. TEG ${ }^{\circ}$ analysis of the individuals was performed on the same day to prevent variations in machine function from day to day. Two samples were run simultaneously in the 2 channels of the TEG ${ }^{\circ}$. Since it has been found that red blood cells influence the mechanical properties of clots due to their viscoelastic properties [26], PRP was used to assess the functioning of the coagulation cascade in the presence of platelets. The citrated PRP $(340 \mu \mathrm{l})$ was added to the oscillating cup and $20 \mu \mathrm{l}$ of calcium chloride $\left(\mathrm{CaCl}_{2}\right)$ added to activate the coagulation process. The process was allowed to run until LY30 was reached. Anticoagulant and antiplatelet medication was not considered a reason to exclude the patient as these treatments do not affect $\mathrm{TEG}^{\circ}$ analysis, since the action of thrombin masks any antithrombotic or antiplatelet activity $[27,28]$.

\section{Statistical analysis}

\section{In SEM to compare fibrin fiber thickness}

The measurements of each participant were done using ImageJ (Version 1,74i, Java) and the values were compared statistically. Statistical analysis was performed on Statistical Analysis System (SAS) using repeated measures analysis of variance (ANOVA). A $p$-value of less than 0.05 was considered significant.

\section{In AFM to compare platelet- and fibrin fiber-elasticity}

The Young's modulus of 50 randomly selected forcedistance curves with good fit on each cell or area of every patient, were compared between the 2 groups (healthy and TIA individuals). SAS software was used to perform the comparison by utilising the generalized mixed model for repeated measures function. A $p$-value of 0.05 was considered statistically significant. These comparisons were performed for platelet- and fibrin fiber-elasticity.

\section{In $T^{\circ} G^{\circ}$ to compare hemostatic profiles of the 2 groups}

The non-parametric Mann-Whitney $U$ test was performed using the SAS software. A two-tailed, one sided test was performed to determine the $p$-value. A $p$-value of less than 0.05 was considered statistically significant.

\section{Results}

\section{Scanning electron microscopy}

SEM is a useful tool for studying morphological alterations of platelets that could be indicative of activation and fibrin fiber arrangement. Red blood cell alterations may affect the density of the clot and the degree of platelet activation, for this reason whole blood was not included in this study.

Figure 1 represents the platelets of healthy- and TIAindividuals. The healthy platelets are spherical with few pseudopodia visible (Fig. 1a) indicated by pink arrows. The presence of numerous pseudopodia, change in shape and spreading are indicative of activation, but is not present or expected in the healthy individuals [29]. Since these healthy individuals are not suffering from any inflammatory condition and no thrombin was added in vitro, inert or slightly activated platelets were expected. Slight activation may be due to contact activation during the preparation of the sample. The healthy platelets are consistent with previously published results [30].

Figure $1 \mathrm{~b}$ to $\mathrm{h}$ represents the platelets of the TIA patients in the presence of metabolic syndrome included in this study. In Fig. 1b and $c$ the typical platelets of the patients can be seen. These platelets are characterized by numerous pseudopodia (pink arrows) and spreading (green arrows) indicating activation. In Fig. 1d, e and $f$ platelet-platelet interactions, also indicative of platelet activity, can be seen. Importantly, platelets wees aggregates are present (as seen in Fig. $1 \mathrm{~g}$ and $\mathrm{h}$ depicted by yellow arrows) in the TIA individuals. The blue arrows in Fig. 1b, c, $d$ and e indicate open canalicular systems (OCSs) that allow granules inside the platelet to exit the platelet.

The fibrin networks of the healthy and TIA individuals were also studied using SEM. As briefly mentioned the fibrin networks of healthy individuals typically consist of thick or major fibers (turquoise arrows) and thin or minor fibers (purple arrows) $[19,20]$. The thick major fibers make up the majority of the clot as shown in Fig. 2a. In contrast the fibrin networks of TIA individuals have an altered appearance as shown in Fig. $2 b$ to $h$ when compared to that of control individuals (Fig. 2a). Fibrin fibers appear less organized with a higher density of 

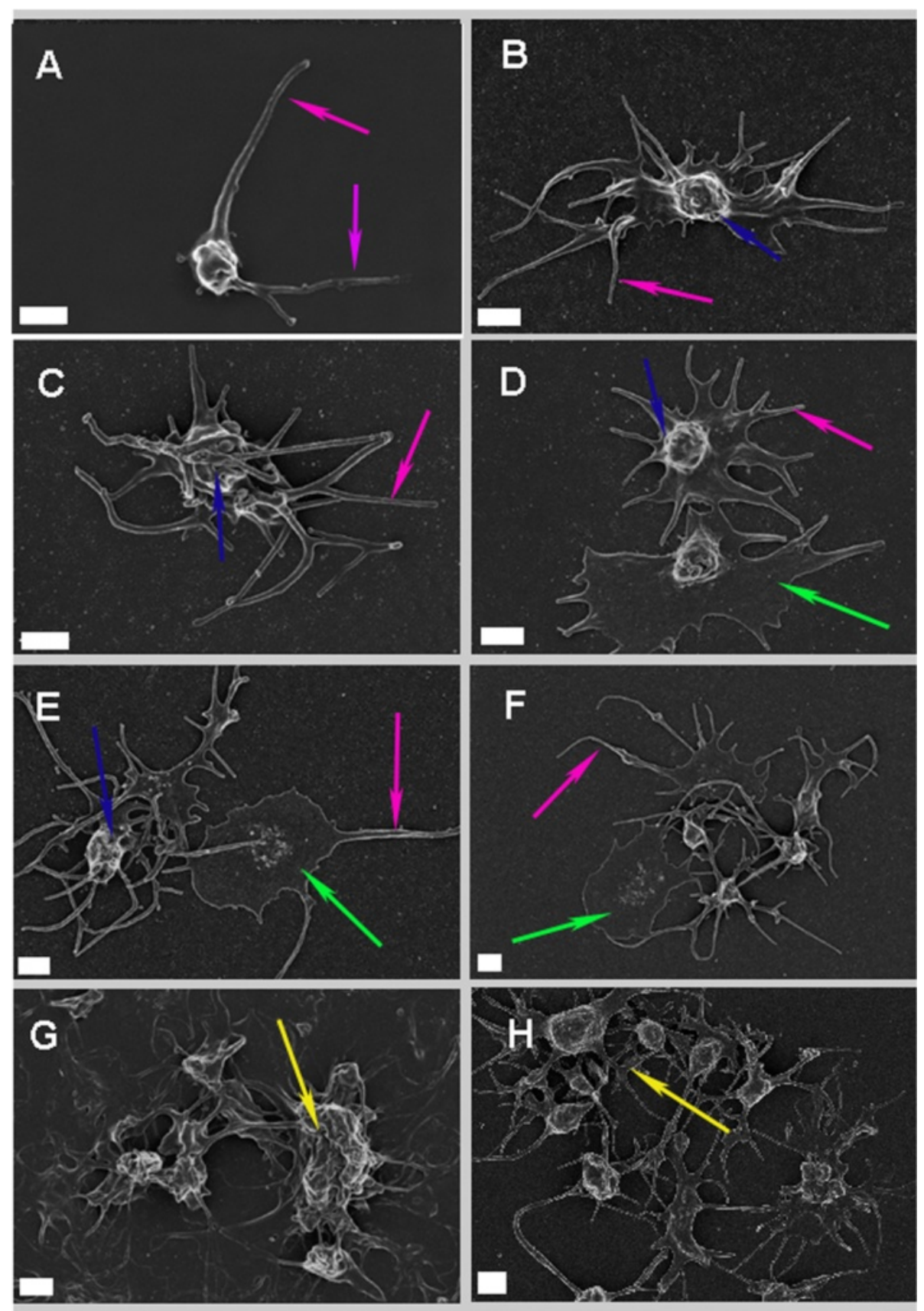

Fig. 1 Platelets of TIA individuals in the presence of metabolic syndrome. a: Platelet of a typical healthy individual. b-e: TIA platelets. f-h: TIA platelet interactions and aggregates (Scale bar: $1 \mu \mathrm{m}$ ). Pink arrows: Pseudopodia. Blue arrows: OCS. Green arrows: Platelet spreading. Yellow arrows: Platelet aggregates

thick major fibers and minor fibers forming a thick net (orange arrows) covering the clot in some individuals (Fig. 2e, $\mathrm{f}$ and $\mathrm{h}$ ). Upon closer examination the fibers are also less taut, folding and twisting (red arrows) forming an irregular web of fibers as seen in Fig. $2 b$ and c. Fibers also appear "sticky" creating thick masses of fibrin fibers (green arrow) as visualized in Fig. 2g.
Measurements of fibrin fiber thickness in the 2 groups were performed and a mean of $98.682 \mathrm{~nm}$ and $164.365 \mathrm{~nm}$ was determined in the control and experimental groups respectively. A $p$-value of $<0.001$ was determined indicating that a statistically significant increase in fibrin fiber thickness was present in the experimental group. 

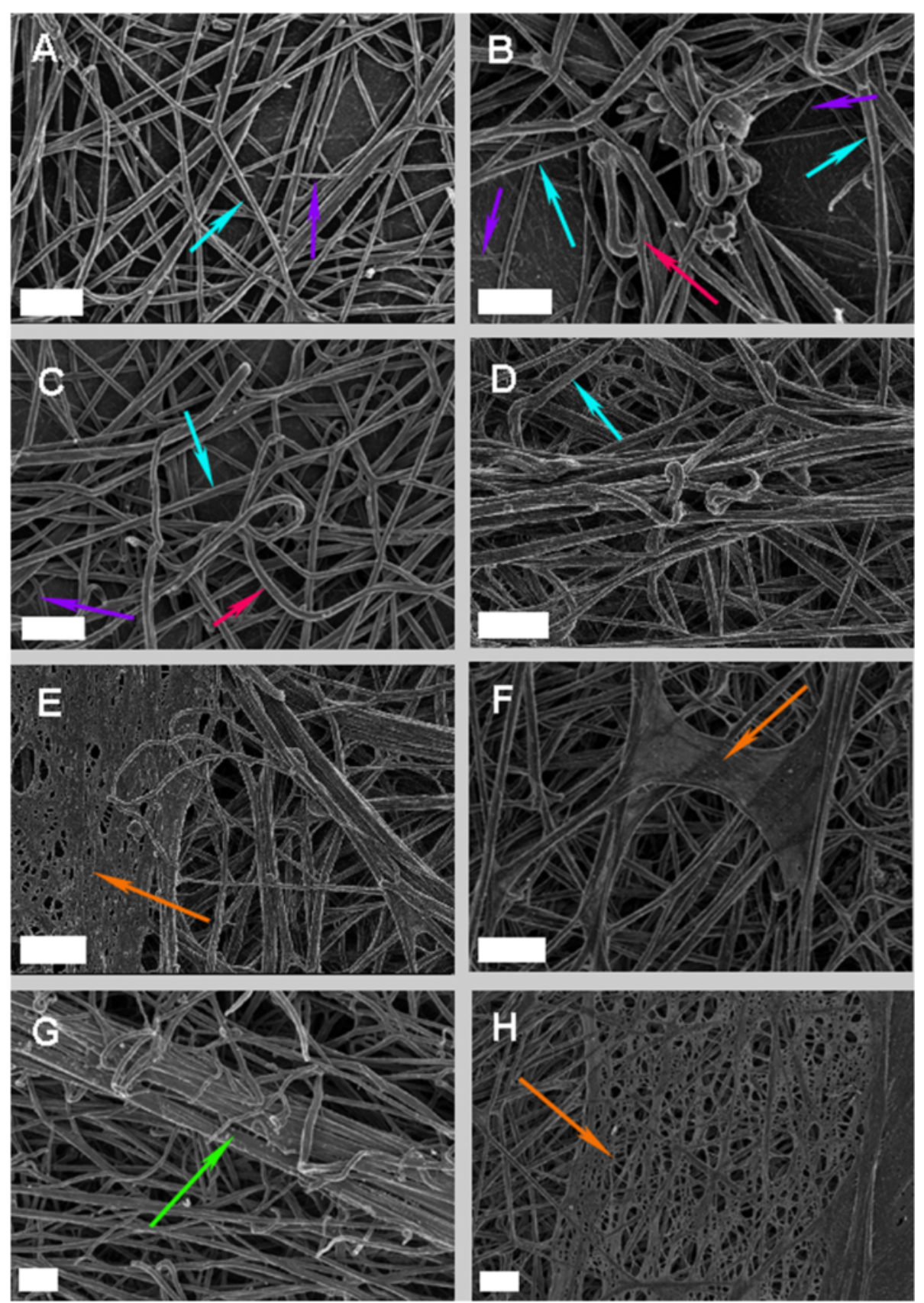

Fig. 2 Fibrin networks of a typical healthy individual and TIA individuals. a: Healthy fibrin network (b-h): Fibrin network of TIA in the presence of metabolic syndrome (Scale bar: $1 \mu \mathrm{m}$ ). Turquoise arrows: Thick major fibers. Purple arrows: Thin minor fibers. Orange arrows: Net-like covering of thin fibers. Red arrows: Bending, less taut fibers. Green arrow: Sticky mass of fibrin fibers

\section{Atomic force microscopy}

Due to the finding on the SEM, AFM was performed on the PRP of the individuals participating in this study to determine whether, in conjunction to the morphological changes, alterations in the nano-mechanical properties of the platelets and fibrin network fibers, that could affect coagulation, were present. Figure 3 represents the images obtained using AFM, indicating that the morphological findings on the AFM correlate with the findings on the SEM micrographs since platelet- and fibrin network-morphology appear similar with both techniques. Figure $3 \mathrm{a}$ represents the platelet of a healthy control individual and Fig. $3 \mathrm{~b}$ the platelet of a TIA individual with activation visible (as seen with the presence of pseudopodia and spreading). Figure $3 \mathrm{c}$ and $\mathrm{d}$ (healthy participants) and $3 \mathrm{~d}$ (experimental group) represent the 

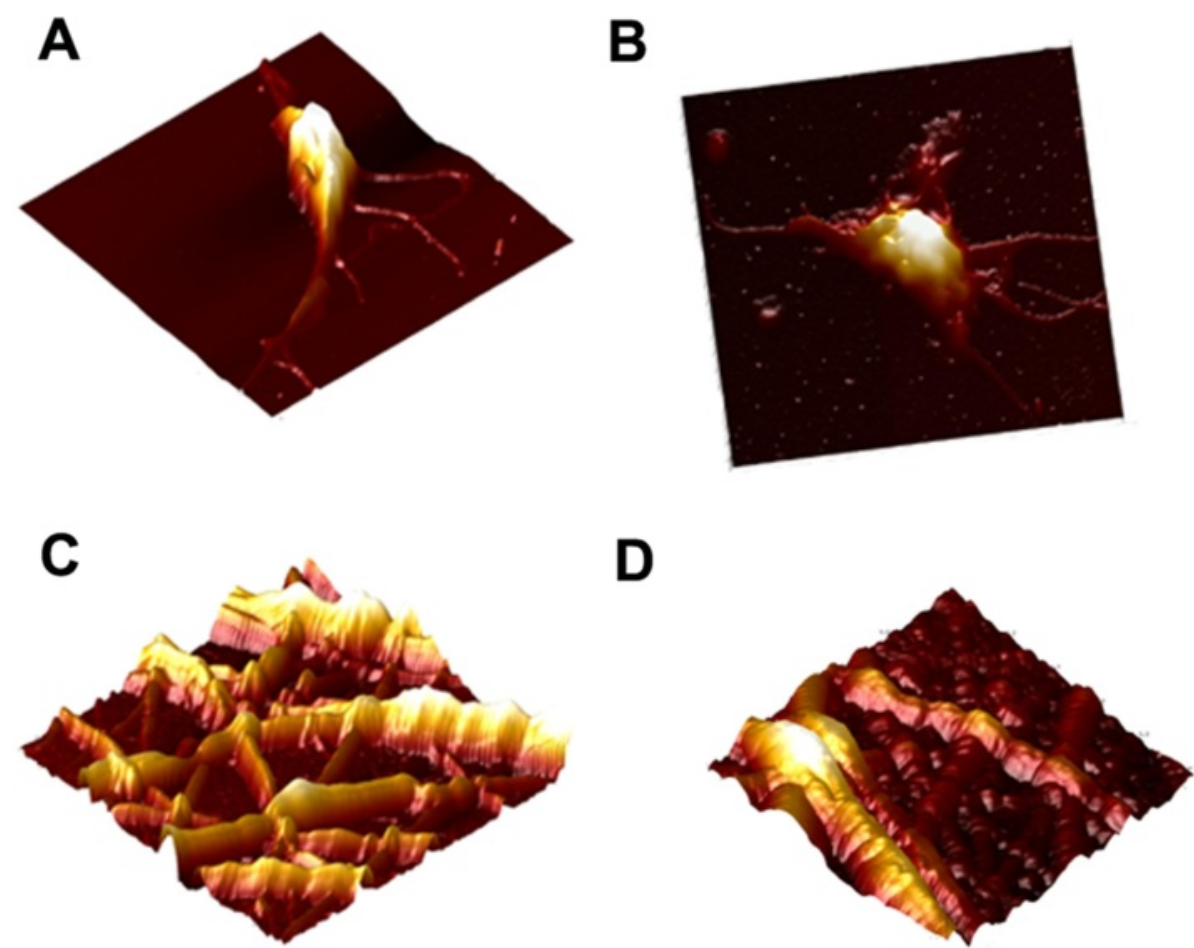

Fig. 3 AFM topography of platelets and fibrin networks. a: Healthy platelet. b: TIA platelet. c: Fibrin network of healthy individual. d: Fibrin network of individual suffering a TIA

fibrin networks of the 2 groups where Fig. 3d shows a denser structure in comparison to the healthy group, similar to that seen using SEM.

Young's modulus, used to measure the nano-mechanical properties of the platelets and fibrin fibers were comparable to results obtained in previous studies [31]. The $p$-values obtained using SAS are represented in Table 3. Young's modulus increased significantly ( $p$-value $<0.05)$ in both the platelets and fibrin networks of the experimental group, indicating that the elasticity of these components decreased in the experimental participants.

\section{Thromboelastography}

The traces obtained from the PRP of the participants are represented in Fig. 4. Figure 4 represents the trace from which reaction time (r-time), k-time, alpha angle

Table 3 The $p$-values obtained from generalized mixed model for repeated measures between the healthy and experimental groups

\begin{tabular}{llll}
\hline Sample & Mean & Median & $p$-value \\
\hline Control platelet & 48.786 & 46.3 & $0.0042^{*}$ \\
TIA platelet & 54.587 & 51.1 & \\
Control fibrin fiber & 12910.84 & 11505 & $0.0007^{*}$ \\
TIA fibrin fiber & 27871.19 & 22263 & \\
\hline
\end{tabular}

* Indicates statistically significant $p$-values
( $\alpha$-angle), maximum amplitude (MA) and clot lysis after $30 \mathrm{~min}$ (LY30) was determined. Figure 4a represents the traces from healthy control individuals and Fig. 4b represents the traces obtained form the experimental group. The traces appear similar in both groups regarding shape and amplitude. Figure $4 c$ and $d$ represent the $\mathrm{v}$-curve from which time to maximum rate of thrombus generation (TMRTG), maximum rate of thrombus generation (MRTG) and total thrombus generation (TTG) were determined. Figure $4 \mathrm{c}$ represents the v-curve obtained from the healthy participants and Fig. 4d the v-curves from the experimental group. No differences could be observed between the participants of the two groups.

In order to determine whether the viscoelastic properties of the clots differed between the healthy and the experimental group, statistical analysis was performed on each parameter measured. The $p$-values obtained in the comparison of the healthy and TIA group are summarized in Table 4 . None of the parameters showed statistically significant differences ( $p$-value less than 0.05) between the two groups. This indicated that both the viscoelastic properties of the clot and the coagulation process were similar in all participants regardless of the presence of metabolic syndrome. LY30 was determined for all the individuals, but in all cases it was found to be $0 \%$, indicating that no clot lysis had taken place $30 \mathrm{~min}$ 

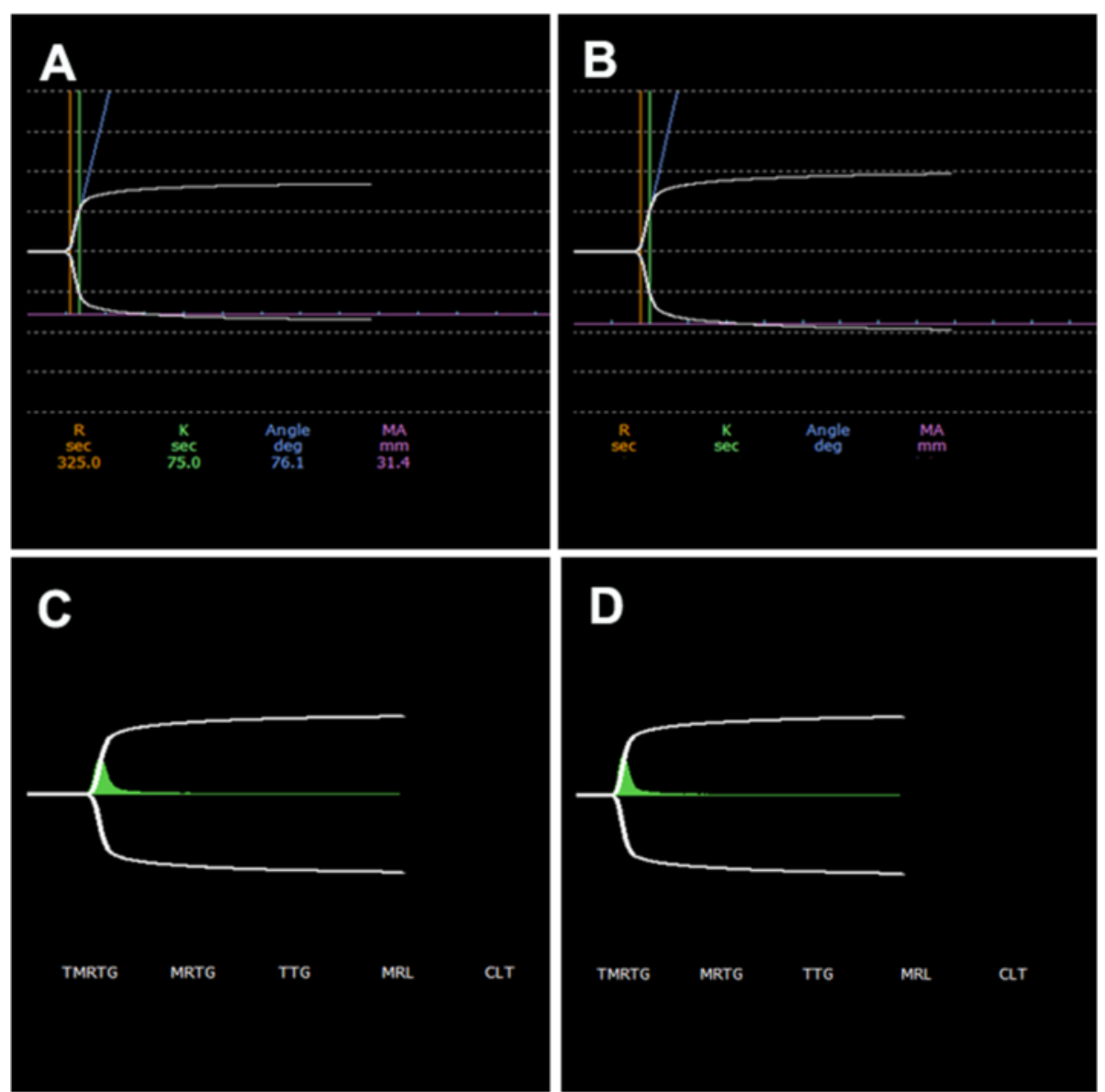

Fig. $4 \mathrm{TEG}^{\circ}$ trace and v-curve obtained from healthy and TIA individuals. a: Trace representative of healthy individuals. b: Trace of TIA individuals. c: v-curve of healthy individual. $\mathbf{d}$ : v-curve of a TIA individual

after maximum amplitude was reached in any of the participants, consistent with normal coagulation.

\section{Discussion}

Platelets are essential in the prevention of blood loss and platelet activation is the first sign of coagulation cascade activation [11]. Platelet shape changes consistent with activation [32] was observed in the experimental group indicating that the coagulation cascade had been activated. This activation occurred spontaneously (without the artificial activation of the coagulation cascade by the addition of thrombin) in the experimental group. The same activation was not present in the experimental group indicating that the coagulation cascade had only been activated in the TIA individuals. These findings confirm the hypothesis of Wu and Hoak in 1975 as well as Hirabayashi and his colleagues in 2004 that platelet activation is closely associated with TIA [33, 34]. Since chronic inflammation is present in these individuals and inflammation is closely associated with activation of the coagulation system, it can be concluded that the activation of platelets occur prior to the cerebral event. This indicates that a procoagulant environment is present in the individuals prior to the onset of the symptoms of the TIA and therefore before formation of the pathological clot. This finding is crucial in the treatment and prevention of TIA since platelet activation is not a result of the ischemic event, but rather plays a role in the development of the attack. Platelet counts were found to be normal in the diseased individuals indicating that the number of platelets did not contribute to the attack, but rather that the activation of the platelets was the crucial step prior to the formation of the clot.

It is well known that platelets increase clot elasticity in normal physiology since they make up approximately $80 \%$ of a thrombus; consequently any alterations in the membrane characteristics (such as elasticity) of the platelets will affect the formed thrombus as well [35]. In the presence of chronic inflammation (as seen in this study) the elasticity of the platelets decreased significantly, giving rise to more rigid platelets with a decreased ability to contract during the final stages of clot 
Table 4 The $p$-values of TEG ${ }^{\bullet}$ parameters obtained with the Mann-Whitney $U$ test

\begin{tabular}{llll}
\hline Parameter & Mean & Median & $p$-value \\
\hline Control r-time & 510 & 502.5 & 0.911 \\
TIA r-time & 480 & 522.5 & \\
Control k-value & 185 & 140 & 0.853 \\
TIA k-value & 137 & 132.5 & \\
Control Alpha(a) angle & 70.58 & 69.15 & 0.436 \\
TIA Alpha(a) angle & 69 & 68.8 & \\
Control MA & 31.62 & 30.25 & 0.190 \\
TIA MA & 37.86 & 35.7 & \\
Control G & 2.42 & 2.15 & 0.165 \\
TIA G & 3.32 & 2.8 & \\
Control MRTGG & 6.532 & 5.645 & 1.000 \\
TIA MRTGG & 6.279 & 6.29 & \\
Control TMRTGG & 9.749 & 9.29 & 0.796 \\
TIA TMRTGG & 9.599 & 10.25 & \\
Control TGG & 276.999 & 245.95 & 0.190 \\
TIA TGG & 469.401 & 313.725 & \\
\hline
\end{tabular}

formation. A decrease in clot retraction, due to decreased platelet contraction, could consequently result in less taut fibers and less organized fibrin networks.

Together with platelets, fibrin fibers make up the majority of the structure of the clot and are the main determinants of the clot mechanics. The fibrin networks of the diseased participants were therefore studied to determine whether alterations in fibrin networks could contribute to changes in clot formation that could lead to the development of a TIA. The fibrin networks of the TIA individuals appeared greatly altered when compared to healthy individuals. These findings are consistent with the findings of other studies that abnormal coagulation plays a major role in ischemic events [36]. The networks appeared denser, less organized with sticky thick fibers that clump together, most likely due to a favouring of lateral aggregation of the protofibrils, this is also consistent with other studies where it was found that denser fibrin networks were associated with a reduction in clot permeability and consequently clot lysis [36-38]. The presence of inflammatory cytokines could further decrease the permeability of the clot as found by Pera et al. in 2015 [39, 40]. Although LY30 measured on the TEG ${ }^{\circ}$ did not indicate hyperfibrinolysis it cannot comment on a decrease in clot fibrinolysis which could be present due to altered fibrin network formation. In some areas the thin fibers form a net that covers large parts of the formed clot. The fibrin fibers of the TIA individuals are significantly thicker than those of the healthy individuals, as seen with statistical analysis. This increase in thickness is most likely due to the propensity of the fibers to stick together as seen in the experimental group. The maximum amplitude (MA) of the clot measured using $\mathrm{TEG}^{\circ}$ corresponds to the speed of fibrin build-up and fibrin cross-linking during clot formation and could therefore be used to study whether the altered fibrin networks affected clot formation. The MA values of the TIA individuals were similar to that of the healthy group. It can therefore be concluded that the formation of the clot was not altered, however the MA does not comment on the quality or thickness of the fibers that could contribute to the formation of the pathological thrombus in the TIA event. Similar to the elasticity of the platelets, the elasticity of the fibrin fibers also decreased (seen with the AFM results) substantiating the theory that a decrease in platelet elasticity will result in a decrease in fibrin fiber elasticity, since clots containing platelets are more elastic than clots without platelets. Less elastic fibers will make it even more difficult for clot retraction to take place explaining the slackness of the fibers seen in the TIA individuals using SEM and AFM. Although fibrinogen concentration may contribute to the formation of a pathological clot, this is probably not the case here, since the rate of clot formation (as measured by $\mathrm{TEG}^{\circ}$ and several plasma based tests) was not significantly different from the healthy controls as would be expected in increased fibrinogen concentrations. Since chronic inflammation is present in the diseased individuals and inflammation activates the coagulation cascade, the changes in the fibrin networks can most likely also be attributed to the continuous inflammatory response that activates platelets. This activation of the platelets, since they function very closely with the fibrin network, is most likely the initiation step of the fibrin network changes seen in the experimental group.

No changes in any of the parameter measured with $\mathrm{TEG}^{\circ}$ could be detected between the healthy control group and the diseased experimental group, indicating that the overall hemostatic profile of the individuals were not affected by metabolic syndrome leading to TIA and that the viscoelastic properties of the clots are similar in the healthy and diseased populations.

Figure 5 and Table 5 represent the findings of this manuscript and how it relates to the development of TIA.

\section{Conclusion}

The findings of this study substantiates the results of other publications which indicate that fibrin network morphology is altered in ischemic events such as stroke [38]. Furthermore it has been postulated that fibrin formation occurs faster in ischemic events in comparison to normal physiology, producing a denser clot (seen with SEM) with increased resistance to fibrinolysis, this study suggests that the quicker fibrin formation and altered fibrin clot properties is a result of the premature 


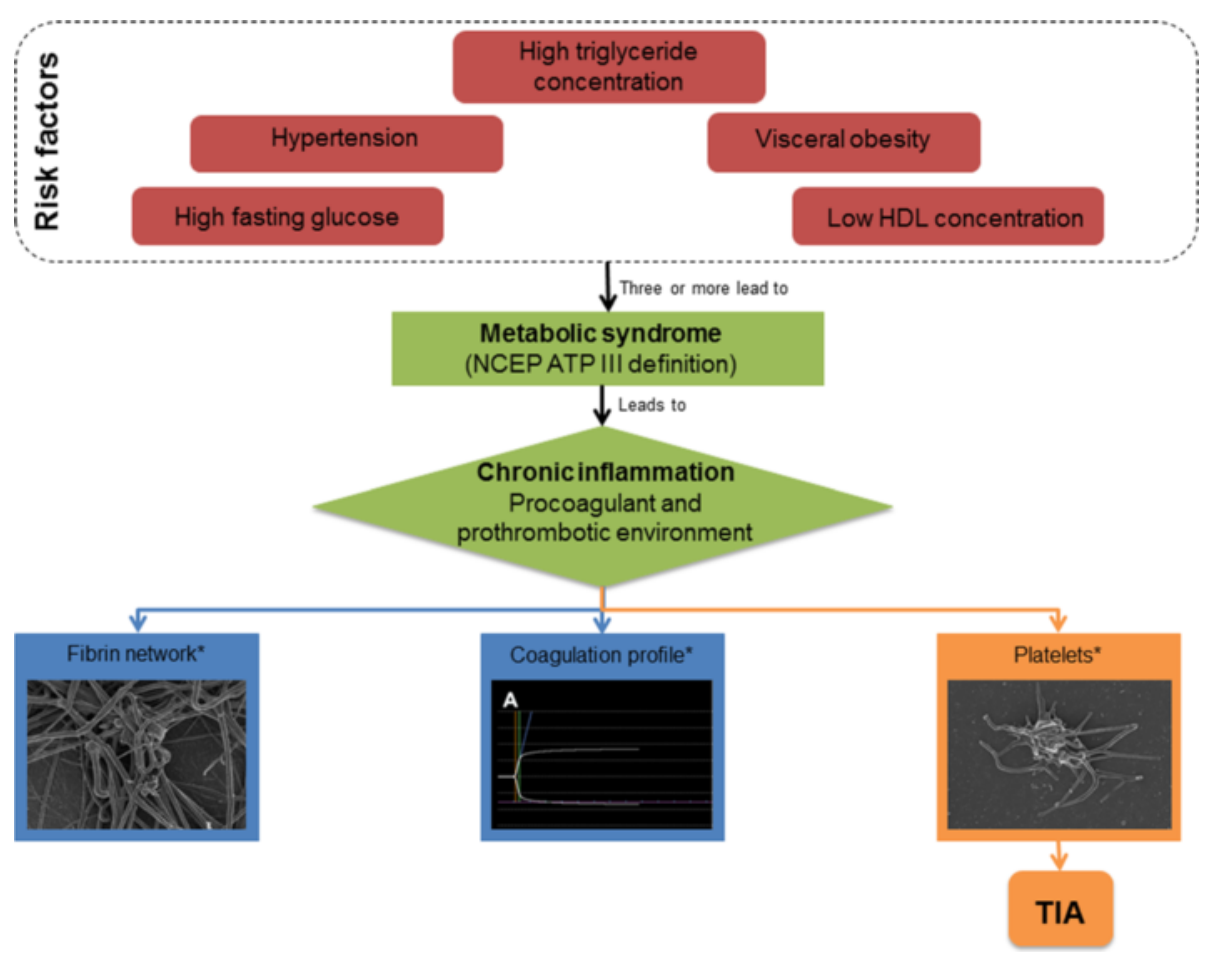

Fig. 5 Figure summarizing the findings of this manuscript. *Findings represented in Table 5. HDL: High-density lipoprotein; TIA: Transient ischemic attack; NCEP ATP III: National cholesterol education program - Adult treatment panel III. Since it is hypothesised that metabolic syndrome causes chronic inflammation that unnecessarily activates the platelets and it is believed that the platelet alterations cause the changes seen in the fibrin networks, this arrow indicates the major culprit in the development of TIA

activation of the coagulation cascade by platelets that are constantly activated in the presence of chronic inflammation as is the case in metabolic syndrome. This is further shown by the findings of the TEG indicating that the coagulation cascade is not altered in the experimental group showing that the cause of the formation of the clot is due to alterations in the hemostatic profile of the participants. Platelet- and fibrin fiberelasticity is decreased resulting in a less permeable clot. Although fibrinolysis may be impeded by the alterations found in the components of coagulation found in this manuscript, fibrinolysis will still occur, leading to the transient period of the symptoms that characterize a TIA.

Table 5 Summary of the findings of this manuscript according to techniques used

\begin{tabular}{|c|c|c|c|}
\hline Technique & Platelets & Fibrin networks & Conclusions drawn \\
\hline \multirow[t]{3}{*}{ SEM } & $\begin{array}{l}\text { Platelet activation present } \\
\text { seen with presence of } \\
\text { pseudopodia and spreading }\end{array}$ & $\begin{array}{l}\text { Denser, less organized fibrin networks in } \\
\text { experimental group }\end{array}$ & $\begin{array}{l}\text { Platelet activation present, altered fibrin network } \\
\text { formation with the addition of thrombin affecting } \\
\text { clot characteristics and possibly the hemostatic } \\
\text { process }\end{array}$ \\
\hline & \multirow[t]{2}{*}{$\begin{array}{l}\text { Platelet aggregation present } \\
\text { in the experimental group }\end{array}$} & $\begin{array}{l}\text { Thick major fibers sticky and clumped } \\
\text { together and thicker than fibers in healthy } \\
\text { individuals }\end{array}$ & \multirow[t]{2}{*}{ These alterations may be due to changes in elasticity } \\
\hline & & $\begin{array}{l}\text { Fibers making up clots of the experimental } \\
\text { group were less taut with visible fibrin fiber } \\
\text { ends not found in the healthy group }\end{array}$ & \\
\hline AFM & $\begin{array}{l}\text { Platelets are less elastic in } \\
\text { the diseased individuals }\end{array}$ & $\begin{array}{l}\text { Fibrin fibers are less elastic in the } \\
\text { experimental group }\end{array}$ & $\begin{array}{l}\text { A decrease in platelet elasticity affects platelet } \\
\text { contraction and therefore clot retraction, resulting } \\
\text { in altered fibrin network elasticity and network } \\
\text { morphology }\end{array}$ \\
\hline $\mathrm{TEG}^{\circ}$ & N/A & N/A & $\begin{array}{l}\text { TEG }{ }^{\circ} \text { was used to determine whether the alterations } \\
\text { obtained with the results of the other techniques } \\
\text { affected the hemostatic profile of the individuals, but } \\
\text { was found not affect the overall coaqulation process }\end{array}$ \\
\hline
\end{tabular}


The findings of this thesis contributes to research and the clinical setting by showing that treatment of a TIA and prevention of future TIA or stroke should focus on the prevention of platelet activation that could prevent to the formation of an abnormal fibrin network and a pathological thrombus.

A limitation to this study is the number of participants; however, this study is adequately powered.

\section{Ethics, consent and permissions}

Ethics approval was obtained from the University of Pretoria Human Ethics Committee. Ethics number: 237/ 2012 with amendment in 2015, new number 24/2015.

\section{Consent to publish}

Written consent to use data and to publish was obtained form each individual participating in this study.

\begin{abstract}
Abbreviations
AFM: Atomic Force Microscopy; ANOVA: Analysis of Variance:

ECG: Electrocardiogram; HDL: High density lipoprotein; HIV: Human Immunodeficiency Virus; HMDS: Hexamethyldisilazane; LY30: Clot lysis 30 min after MA; MA: Maximum Amplitude; MRTG: Maximum Rate of Thrombus Generation; NCEP ATP III: National Cholesterol Education Program, Adult Treatment Panel III; OCS: Open Canalicular System; $\mathrm{OsO}_{4}$ : Osmium Tetroxide; PB: Phosphate Buffer; PRP: Platelet-rich Plasma; QNM ${ }^{\mathrm{T} w}$ : Quantitative Nanomechanical Property Mapping ${ }^{\text {TM}}$; r-time: Reaction Time; SANBS: South African National Blood Service; SAS: Statistical Analysis System; SEM: Scanning Electron Microscopy; TEG: Thromboelastography; TMRTG: Time to Maximum Rate of Thrombus Generation; TIA: Transient Ischemic Attack; TTG: Total Thrombus Generation.
\end{abstract}

\section{Competing interests}

The authors declare that they have no competing interests.

\section{Authors' contributions}

MvR, WD and EP contributed to the study design. MvR collected the samples, carried out the microscopy and $\mathrm{TEG}^{\circ}$ studies, interpreted the data and drafted the manuscript. WD aided in the collection of the data and the diagnosis and recruitment of the patients. EP critically revised the manuscript prior to submission. EP contributed funding under a grant received from the National Research Foundation. AVB aided in AFM analyses; RE performed statistical analysis and aided in interpretation. All authors gave final approval for publication.

\section{Funding}

National Research Foundation (NRF) South Africa: E Pretorius.

\section{Author details}

'Department of Physiology, Faculty of Health Sciences, University of Pretoria, Private Bag x323, Arcadia 0007, South Africa. ${ }^{2}$ Department of Neurology, Faculty of Health Sciences, University of Pretoria, Arcadia 0007, South Africa. ${ }^{3}$ Department of Statistics, Faculty of Natural Sciences, University of Pretoria, Arcadia 0007, South Africa. ${ }^{4}$ Unit of Microscopy and Microanalysis, Faculty of Natural Sciences, Arcadia 0007, South Africa.

Received: 8 May 2015 Accepted: 18 June 2015

Published online: 04 July 2015

\section{References}

1. Kernan WN, Ovbiagele B, Black HR, Bravata DM, Chimowitz MI, Ezekowitz $M D$, et al. Guidelines for the prevention of stroke in patients with stroke and transient ischemic attack: a guideline for healthcare professionals from the american heart association/american stroke association. Stroke. 2014;45(7):2160-236
2. Depta JP, Bhatt DL. New approaches to inhibiting platelets and coagulation. Ann Rev Pharmacol Toxicol. 2015;55:373-97.

3. Albers GW, Caplan LR, Easton JD, Fayad PB, Mohr J, Saver JL, et al. Transient ischemic attack - proposal for a new definition. New Engl J Med. 2002;347(21):1713.

4. Shah KH, Edlow JA. Transient ischemic attack: review for the emergency physician. Ann Emerg Med. 2004;43(5):592-604.

5. Sonni $\mathrm{S}$, Thaler DE. Transient ischemic attack: omen and opportunity. Clevel Clin J Med. 2013;80(9):566-76.

6. van Rooy MJ, Pretorius E. Metabolic syndrome, platelet activation and the development of transient ischemic attack or thromboembolic stroke. Thromb Res. 2015;135(3):434-42.

7. Kassi E, Pervanidou P, Kaltsas G, Chrousos G. Metabolic syndrome: definitions and controversies. BMC Med. 2011:9:48.

8. Murray KN, Girard S, Holmes WM, Parkes LM, Williams SR, Parry-Jones AR, et al. Systemic inflammation impairs tissue reperfusion through endothelindependent mechanisms in cerebral ischemia. Stroke. 2014;45(11):3412-9.

9. Pober JS, Sessa WC. Inflammation and the blood microvascular system. Cold Spring Harbor. 2015;7(1):a016345.

10. Fowler A, Perry DJ. Laboratory monitoring of haemostasis. Anaesthesia. 2015;70 Suppl 1:68-72.

11. Davi G, Patrono C. Platelet activation and atherothrombosis. New Engl J Med. 2007;357(24):2482-94.

12. Lam WA, Chaudhuri O, Crow A, Webster KD, Li TD, Kita A, et al. Mechanics and contraction dynamics of single platelets and implications for clot stiffening. Nature. 2011;10(1):61-6.

13. Roska FJ, Ferry JD. Studies of fibrin film. I. Stress relaxation and birefringence. Biopolymers. 1982;21(9):1811-32.

14. Weisel JW. Structure of fibrin: impact on clot stability. J Thromb Haemost. 2007;5 Suppl 1:116-24

15. Ott I. Soluble tissue factor emerges from inflammation. Circ Res. 2005;96(12):1217-8.

16. Biswas S, Ghoshal PK, Mandal SC, Mandal N. Relation of anti- to proinflammatory cytokine ratios with acute myocardial infarction. Kor J Internal Med. 2010;25(1):44-50.

17. Demetz G, Ott I. The Interface between inflammation and coagulation in cardiovascular disease. Int J Inflamm. 2012;2012:860301.

18. Lipinski S, Bremer L, Lammers T, Thieme F, Schreiber S, Rosenstiel P. Coagulation and inflammation. Molecular insights and diagnostic implications. Hamostaseologie. 2011;31(2):94-102.

19. Pretorius E, Oberholzer HM, Vieira WA, Smit E. Ultrastructure of platelets and fibrin networks of asthmatic mice exposed to selenium and Withania somnifera. Anat Sci Int. 2009;84(3):210-7.

20. Pretorius $\mathrm{E}$, Vieira W, Oberholzer $\mathrm{H}$, Auer R. Comparative scanning electron microscopy of platelets and fibrin networks of humans and different animals. Int J Morphol. 2009;27(1):69-76.

21. Pittenger B, Erina N, Su C. Quantitative mechanical property mapping at the nanoscale with PeakForce QNM. Application Note Veeco Instruments Inc 2010.

22. Berquand A. Quantitative imaging of living biological samples by Peak Force QNM Application Note Veeco Instruments Inc 2011.

23. Dulińska I, Targosz M, Strojny W, Lekka M, Czuba P, Balwierz W, et al. Stiffness of normal and pathological erythrocytes studied by means of atomic force microscopy. J Biochem Biophys Method. 2006;66(1):1-11.

24. Kolar P, Tomankova K, Malohlava J, Zapletalova J, Vujtek M, Safarova K, et al. The effect of photodynamic treatment on the morphological and mechanical properties of the HeLa cell line. Gen Physiol Biophys. 2013:32(3):337-46.

25. Derjaguin B, Muller V, Toporov YP. Effect of contact deformations on the adhesion of particles. J Colloid Interf Sci. 1975;53(2):314-26.

26. Gersh KC, Nagaswami C, Weisel JW. Fibrin network structure and clot mechanical properties are altered by incorporation of erythrocytes. Thromb Haemost. 2009;102(6):1169-75.

27. Wegner J, Popovsky MA. Clinical utility of thromboelastography: one size does not fit all. Semin Thromb Haemost. 2010;36(7):699-706.

28. Lang T, Von Depka M. Possibilities and limitations of thrombelastometry/ graphy. Hamostaseologie. 2006;23 Suppl 1:S20-9.

29. Zucker M, Nachmias V. Platelet activation. Arterioscler Thromb Vasc Biol. 1985;5(1):2-18

30. Pretorius E, Oberholzer HM, Smit E, Steyn E, Briedenhann S, Franz CR. Ultrastructural changes in platelet aggregates of HIV patients: a scanning electron microscopy study. Ultrastruct Pathol. 2008;32(3):75-9. 
31. Du Plooy JN, Buys A, Duim W, Pretorius E. Comparison of platelet ultrastructure and elastic properties in thrombo-embolic ischemic stroke and smoking using atomic force and scanning electron microscopy. PLoS One. 2013;8(7):e69774

32. Kuwahara M, Sugimoto M, Tsuji S, Matsui H, Mizuno T, Miyata S, et al. Platelet shape changes and adhesion under high shear flow. Arterioscler Thromb Vasc Biol. 2002;22(2):329-34.

33. Wu KK, Hoak JC. Increased platelet aggregates in patients with transient ischemic attacks. Stroke. 1975;6(5):521-4.

34. Hirabayashi H, Shimizu M, Kohara S, Shinohara Y. Activated platelets in transient global amnesia and TIA. Neurology. 2004;63(4):736-8.

35. Carr Jr ME, Carr SL. Fibrin structure and concentration alter clot elastic modulus but do not alter platelet mediated force development. Blood Coag Fibrinol. 1995;6(1):79-86.

36. Stanford SN, Sabra A, D'Silva L, Lawrence M, Morris RH, Storton S, et al. The changes in clot microstructure in patients with ischaemic stroke and the effects of therapeutic intervention: a prospective observational study. BMC Neurol. 2015;15:35

37. Undas A, Podolec P, Zawilska K, Pieculewicz M, Jedlinski I, Stepien E, et al. Altered fibrin clot structure/function in patients with cryptogenic ischemic stroke. Stroke. 2009;40(4):1499-501.

38. Undas A, Slowik A, Wolkow P, Szczudlik A, Tracz W. Fibrin clot properties in acute ischemic stroke: relation to neurological deficit. Thromb Res. 2010;125(4):357-61.

39. Pera J, Undas A, Topor-Madry R, Jagiella J, Klimkowicz-Mrowiec A, Slowik A Fibrin clot properties in acute stroke: what differs cerebral hemorrhage from cerebral ischemia? Stroke. 2012;43(5):1412-4.

40. Wolberg AS. Plasma and cellular contributions to fibrin network formation, structure and stability. Haemophilia. 2010;16 Suppl 3:7-12.

41. Flier JS, Underhill LH, Furie B, Furie BC. Molecular and cellular biology of blood coagulation. New Engl J Med. 1992;326(12):800-6.

42. Angiolillo DJ, Ueno M, Goto S. Basic principles of platelet biology and clinical implications. Circ J. 2010;74(4):597-607.

43. Moser L, Callahan KS, Cheung AK, Stoddard GJ, Munger MA. ACE inhibitor effects on platelet function in stages I-II hypertension. J CardiovasC Pharmacol. 1997;30(4):461-7.

44. Serdynska-Szuster M, Banaszewska B, Spaczynski R, Pawelczyk L. Effects of metformin therapy on markers of coagulation disorders in hyperinsulinemic women with polycystic ovary syndrome. Ginekol Pol. 2011;82(4):259-64.

45. Stegenga ME, van der Crabben SN, Levi M, de Vos AF, Tanck MW, Sauerwein HP, et al. Hyperglycemia stimulates coagulation, whereas hyperinsulinemia impairs fibrinolysis in healthy humans. Diabetes. 2006;55(6):1807-12.

46. Stockmans F, Deberdt W, Nystrom A, Nystrom E, Stassen J, Vermylen J, et al. Inhibitory effect of piracetam on platelet-rich thrombus formation in an animal model. Thromb Haemost. 1998:79:222-7.

\section{Submit your next manuscript to BioMed Central and take full advantage of:}

- Convenient online submission

- Thorough peer review

- No space constraints or color figure charges

- Immediate publication on acceptance

- Inclusion in PubMed, CAS, Scopus and Google Scholar

- Research which is freely available for redistribution 